\title{
CARACTERIZACIÓN FÍSICA-QUÍMICA-ENERGÉTICA DE PELLETS DE HOJARASCA RESIDUAL COMO BIOCOMBUSTIBLE RENOVABLE
}

\section{PHYSICAL-CHEMICAL-ENERGY CHARACTERIZATION OF WASTE FALLEN LEAVES PELLETS AS A RENEWABLE BIOFUEL}

\author{
William A. González*, Diana López**, Juan F. Pérez* \\ * Universidad de Antioquia, Facultad de ingeniería, Grupo GIMEL. \\ Calle 67 \# 53-10, Medellín, Antioquia, Colombia. \\ Teléfono: +5742195550 \\ E-mail: williama.gonzalez@udea.edu.co \\ jfernando.perez@udea.edu.co \\ ** Universidad de Antioquia, Facultad de ciencias exactas y naturales, Grupo QUIREMA. \\ Calle 67 \# 53-10, Medellín, Antioquia, Colombia. \\ Teléfono: +57 42196614 \\ E-mail: diana.lopez@udea.edu.co
}

Resumen: En el campus central de la Universidad de Antioquia se generan alrededor de 2.8 ton/mes de hojarasca (base seca). El objetivo de este trabajo es evaluar este material residual como alternativa energética mediante la estimación de su potencial energético y la caracterización física y termoquímica de la biomasa residual sometida a un pretratamiento de densificación (peletizado). Para la obtención del combustible se utilizó una peletizadora a escala de laboratorio $(30-40 \mathrm{~kg} / \mathrm{h}$ ), para la preparación de los pellets se evaluó el contenido de humedad y de glicerol como agentes aglomerantes dentro de la mezcla. El material densificado posee una densidad energética entre $5500-7300 \mathrm{MJ} / \mathrm{m} 3$ frente a $2800 \mathrm{MJ} / \mathrm{m} 3$ de la hojarasca sin pretratamiento. El aumento de este parámetro muestra un mejoramiento de las propiedades energéticas del combustible para ser usado en procesos termoquímicos. Con el aumento del contenido de humedad se encuentra una disminución del $15 \%$ en la cantidad de material volátil del combustible. Evaluando las propiedades físicas se encuentra que la resistencia a la tracción de los pellets varía entre 220-240 kPa y que con el aumento del contenido de glicerol los pellets tienen una mayor capacidad de deformación (deformaciones entre 0.5-2.25 mm), lo que se relaciona con una mayor durabilidad de los pellets.

Palabras clave: Hojarasca, densificación, pellets, residuos a energía, valoración energética.

\begin{abstract}
The University of Antioquia main campus generates about 2.8 ton/month of fallen leaves (dry basis). The objective of this work is to evaluate this waste material as an energy alternative by estimating the energy potential and the physical and thermochemical characterization of the residual biomass subjected to a densification pretreatment (pelletizing process). To obtain the fuel a pelletizer was used on a laboratory scale $(30-40 \mathrm{~kg} / \mathrm{h})$. For the preparation of the pellets the moisture and glycerol content were evaluated as binder agents within the mixture. The densified material has an energy density between $5500-7300 \mathrm{MJ} / \mathrm{m}^{3}$ versus $2800 \mathrm{MJ} / \mathrm{m} 3$ of fallen leaves without
\end{abstract}


pretreatment. The increase of this parameter shows an improvement of the energy properties of the fuel to be used in thermochemical processes. The fuel volatile matter decrease $15 \%$ when the moisture content increase. Evaluating the physical properties it was found that the tensile strength of the pellets is between $220-240 \mathrm{kPa}$ and that with the increase of the glycerol content the pellets have a greater capacity of deformation (deformations between $0.5-2.25 \mathrm{~mm}$ ), which indicates a greater durability of the pellets.

Keywords: Fallen leaves, densification, pellets, waste to energy, energy use.

\section{INTRODUCCIÓN}

El aumento de la producción de residuos se asocia con el crecimiento de la población (Medina Jimenez et al., 2017). La producción de desechos en Medellín es de aproximadamente 1800 ton/día, con una tasa de generación per cápita de alrededor de $0.45 \mathrm{~kg} /$ habitante/día. La producción total de desechos debido a zonas verdes y jardines es de aproximadamente $4.5 \%$. Por lo tanto, una de las alternativas renovables de mayor potencial es el uso de biomasa residual como fuente de energía (Larochelle et al., 2012). El uso de hojarasca obtenida a partir de residuos municipales como materia prima para la conversión energía aumenta el valor de los desechos y reduce el impacto ambiental asociado a su eliminación y emisiones de CH4 y CO2 (García-Maraver et al., 2012)(William et al. , 2015). Generalmente, las ciudades recogen hojas caídas para quemarlas o depositarlas en rellenos sanitarios (Criollo, 2014), aunque en muchos casos se usan en procesos de compostaje para generar un fertilizante orgánico a partir de la descomposición de la materia prima y la liberación de gases volátiles (Piepenschneider et al., 2016)(Bhange et al., 2012). El proceso de compostaje tiene un factor de emisión de aproximadamente 95-265 gCO2/kgresiduos, mientras que los procesos de conversión termoquímicos de biomasa generan alrededor de 80 $\mathrm{gCO} 2 /$ kgresiduos de residuos, es decir, hay una reducción entre el $15 \%$ y el $70 \%$ de las emisiones contaminantes si la hojarasca residual se tratan en procesos de generación de energía en lugar de compostaje (Andersen et al., 2010)(Zhang et al., 2000).

Los jardines del campus principal de la Universidad de Antioquia (Colombia) producen alrededor de 2.8 toneladas de hojarasca seca por mes. Este desecho se utiliza en la Universidad para producir compostaje, que se utiliza como fertilizante en los jardines del campus. Actualmente, se está generando un exceso de abono orgánico, por lo que la Universidad ha decidido contratar empresas externas para recolectar y disponer los residuos forestales generados (Figura 1). En el presente trabajo se realiza un estudio de factibilidad técnica de la densificación de hojarasca residual para aplicaciones energéticas a través de conversión termoquímica.
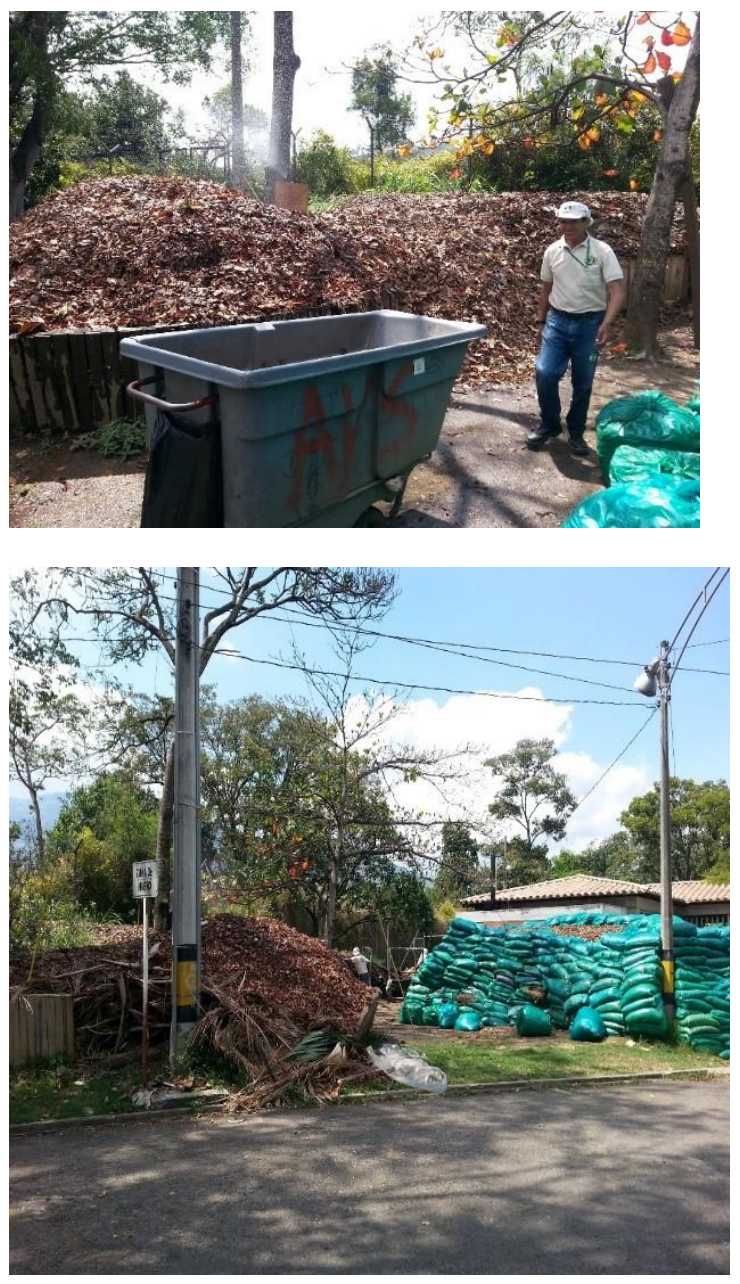

Figura 1. Hojarasca residual apilada para compostaje en la Universidad de Antioquia 
Tabla 1. Propiedades físicas de los pellets

\begin{tabular}{|c|c|c|c|c|c|c|c|}
\hline Pellet & $\begin{array}{c}\text { Durez } \\
\text { a } \\
\text { [Shore } \\
\text { D] }\end{array}$ & $\begin{array}{c}\rho\left[\mathbf{k g} \cdot \mathbf{m}^{-}\right. \\
\left.{ }^{-}\right]\end{array}$ & $\rho_{p}\left[\mathrm{~kg} \cdot \mathrm{m}^{-3}\right]$ & $\begin{array}{l}\mathbf{P} \\
\mathbf{F} \\
{[-]}\end{array}$ & $\begin{array}{c}\text { Deq } \\
{[\mathrm{mm}]}\end{array}$ & $\Psi[-]$ & $\begin{array}{c}\mathbf{S} / \mathbf{V} \\
{[\mathbf{m m}} \\
\left.{ }^{-1}\right]\end{array}$ \\
\hline $\begin{array}{l}\text { M10- } \\
\text { G00 }\end{array}$ & $\begin{array}{c}49.0 \pm 4 \\
.3\end{array}$ & $524.7 \pm 5.6$ & $\begin{array}{c}1102.07 \pm 32 \\
2\end{array}$ & $\begin{array}{l}0 . \\
48\end{array}$ & $14.4 \pm 1.6$ & $\begin{array}{c}0.78 \pm 0.0 \\
4\end{array}$ & $\begin{array}{c}2.36 \\
\pm 0.6 \\
4\end{array}$ \\
\hline $\begin{array}{l}\text { M15- } \\
\text { G00 }\end{array}$ & $\begin{array}{c}27.8 \pm 2 \\
.0\end{array}$ & $\begin{array}{c}477.6 \pm 10 . \\
6\end{array}$ & $\begin{array}{c}1076.72 \pm 35 \\
7\end{array}$ & $\begin{array}{l}0 . \\
44\end{array}$ & $12.7 \pm 0.9$ & $\begin{array}{c}0.84 \pm 0.0 \\
2\end{array}$ & $\begin{array}{c}1.60 \\
\pm 0.3 \\
0\end{array}$ \\
\hline $\begin{array}{l}\text { M20- } \\
\text { G00 }\end{array}$ & $\begin{array}{c}29.8 \pm 4 \\
.4\end{array}$ & $\begin{array}{c}452.7 \pm 13 \\
8\end{array}$ & $\begin{array}{c}1029.81 \pm 20 \\
6\end{array}$ & $\begin{array}{l}0 . \\
44\end{array}$ & $14.5 \pm 1.2$ & $\begin{array}{c}0.80 \pm 0.0 \\
3\end{array}$ & $\begin{array}{c}2.10 \\
\pm 0.3 \\
5\end{array}$ \\
\hline $\begin{array}{l}\text { M10- } \\
\text { G05 }\end{array}$ & $\begin{array}{c}25.2 \pm 3 \\
.2\end{array}$ & $461.5 \pm 9.0$ & $980.21 \pm 8.7$ & $\begin{array}{l}0 . \\
47\end{array}$ & $12.5 \pm 1.1$ & $\begin{array}{c}0.84 \pm 0.0 \\
3\end{array}$ & $\begin{array}{c}1.51 \\
\pm 0.3 \\
8\end{array}$ \\
\hline $\begin{array}{l}\text { M15- } \\
\text { G05 }\end{array}$ & $\begin{array}{c}18.0 \pm 2 \\
.3\end{array}$ & $420.4 \pm 2.6$ & $946.47 \pm 20.1$ & $\begin{array}{l}0 . \\
44\end{array}$ & $13.1 \pm 0.8$ & $\begin{array}{c}0.84 \pm 0.0 \\
2\end{array}$ & $\begin{array}{c}1.48 \\
\pm 0.1 \\
9\end{array}$ \\
\hline $\begin{array}{l}\text { M20- } \\
\text { G05 }\end{array}$ & $\begin{array}{c}22.2 \pm 2 \\
.0\end{array}$ & $\begin{array}{c}394.6 \pm 10 . \\
8\end{array}$ & $942.03 \pm 25.4$ & $\begin{array}{l}0 . \\
42\end{array}$ & $14.9 \pm 1.1$ & $\begin{array}{c}0.79 \pm 0.0 \\
3\end{array}$ & $\begin{array}{c}2.07 \\
\pm 0.4 \\
0\end{array}$ \\
\hline $\begin{array}{l}\text { M10- } \\
\text { G10 }\end{array}$ & $\begin{array}{c}17.2 \pm 1 \\
.7\end{array}$ & $370.1 \pm 6.0$ & $887.16 \pm 17.1$ & $\begin{array}{l}0 . \\
42\end{array}$ & $\begin{array}{c}13.5 \pm 0.7 \\
5\end{array}$ & $\begin{array}{c}0.84 \pm 0.0 \\
2\end{array}$ & $\begin{array}{c}1.53 \\
\pm 0.2 \\
2\end{array}$ \\
\hline $\begin{array}{l}\text { M15- } \\
\text { G10 }\end{array}$ & $\begin{array}{c}18.7 \pm 2 \\
.1\end{array}$ & $430.1 \pm 7.1$ & $948.51 \pm 29.6$ & $\begin{array}{l}0 . \\
45\end{array}$ & $13.8 \pm 1.1$ & $\begin{array}{c}0.82 \pm 0.0 \\
3\end{array}$ & $\begin{array}{c}1.68 \\
\pm 0.3 \\
1\end{array}$ \\
\hline $\begin{array}{l}\text { M20- } \\
\text { G10 }\end{array}$ & $\begin{array}{c}22.5 \pm 1 \\
.9\end{array}$ & $406.6 \pm 4.8$ & $923.19 \pm 9.1$ & $\begin{array}{l}0 . \\
44\end{array}$ & $15.8 \pm 1.1$ & $\begin{array}{c}0.77 \pm 0.0 \\
3\end{array}$ & $\begin{array}{c}2.39 \\
\pm 0.4 \\
2\end{array}$ \\
\hline
\end{tabular}

\section{METODOLOGÍA}

Las etapas para desarrolladas en el método de trabajo se describen a continuación:

Aforo: debido a las estaciones climáticas de Medellín, se decidió realizar mediciones en los meses de mayor precipitación y de menor brillo solar (mayo y octubre) y los meses de menor precipitación y mayor brillo solar (enero y julio). La medición permite determinar la cantidad de hojarasca producida en la universidad y su contenido de humedad. Además, las muestras de hojarasca se recolectan de manera aleatoria en cada temporada. Las muestras recolectadas se muelen, se mezclan y se secan a temperatura ambiente para la caracterización de la biomasa y la producción del combustible densificado.

Preparación y caracterización de los gránulos: la preparación de gránulos se basa en el diseño experimental estadístico multifactorial $3^{2}$. El contenido de humedad es el primer parámetro experimental. Los tres niveles del contenido de humedad (base masa) son: $10 \%, 15 \%$ y $20 \%$. El segundo factor considerado en el diseño experimental es el contenido de glicerina (base masa) como aglutinante. Para este factor, se seleccionan tres niveles $(0 \%, 5 \%$ y $10 \%)$. La producción de pellets se realizó con una máquina peletizadora de rodillos de $3,75 \mathrm{~kW}$, con un diámetro nominal de orificios de $8 \mathrm{~mm}$, y con una capacidad de $30-40 \mathrm{~kg} / \mathrm{h}$.

Los pellets producidos se caracterizan por análisis físicos y termoquímicos para analizar su calidad como biocombustible sólido. Las propiedades físicas analizadas son: densidades aparente y de partícula, factor de empaquetamiento, diámetro equivalente, esfericidad, relación superficie/volumen, dureza, resistencia a la tracción, durabilidad y microscopía electrónica de barrido (SEM). El análisis termoquímico se realizó mediante análisis próximo, último y bioquímico, reactividad, poder calorífico y densidad de energía.

\section{RESULTADOS}

La Tabla 1 muestra las propiedades físicas de diferentes pellets. La densidad aparente disminuye con el aumento de los contenidos de humedad y glicerol. Una tendencia similar se muestra en la densidad de la partícula y el factor de empaquetamiento. El diámetro equivalente, la esfericidad y la relación superficie/volumen evalúan la relación de aspecto de los pellets, por lo tanto, un pellet con una forma cilíndrica más definida tiene un diámetro equivalente mayor y una relación superficie/volumen, y además una esfericidad menor.

La resistencia a la tracción de los pellets se encuentra entre $220-240 \mathrm{kPa}$, se obtuvo a partir de la fuerza de compresión. La Figura 2 muestra que con el aumento del contenido de humedad y glicerol hay un aumento en la resistencia a la tracción. También es evidente que, con el aumento en el contenido de glicerol, los pellets tienen una mayor capacidad de deformación.

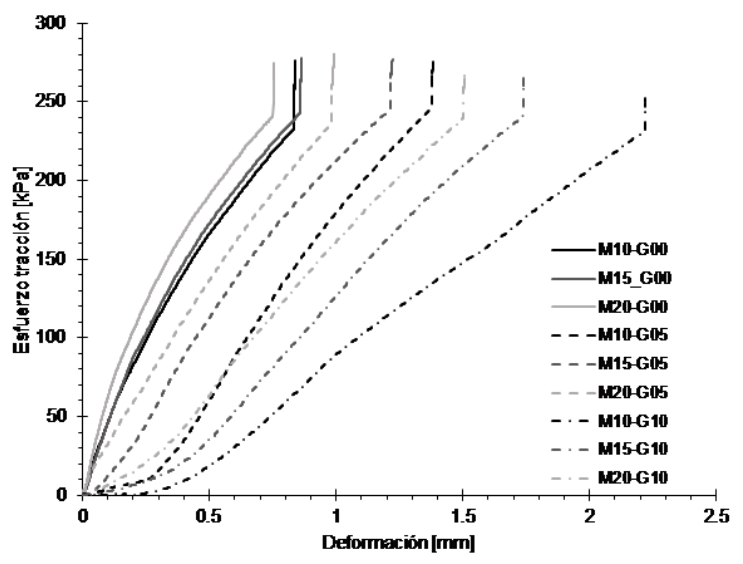

Figura 2. Esfuerzo a tracción 
El análisis próximo muestra variaciones significativas con el aumento en el contenido de humedad (ver Tabla 2). El contenido de material volátil disminuye, mientras que el contenido de carbono fijo aumenta (García, 2015). Este comportamiento se debe al hecho de que al aumentar el contenido de humedad se requiere un aumento en la temperatura de la biomasa para alcanzar la densificación, esto implica un mayor número de recirculaciones de la materia prima en la pelletizadora. Por lo tanto, la liberación de gases volátiles se ve favorecida durante el proceso de densificación. El nivel de calentamiento de las muestras de biomasa aumenta a un mayor contenido de glicerol.

Tabla 2. Análisis proximo y poder calorífico superior

\begin{tabular}{|c|c|c|c|c|c|}
\hline \multirow[t]{2}{*}{ Fuel } & \multicolumn{3}{|c|}{$\begin{array}{c}\text { Análisis próximo } \\
\text { [\% wt. d.b] }\end{array}$} & \multirow[t]{2}{*}{$\begin{array}{l}\text { Humed } \\
\text { ad [\%] }\end{array}$} & \multirow{2}{*}{$\begin{array}{c}\mathrm{PCS} \\
{[\mathrm{MJ} \cdot \mathrm{k}} \\
\mathrm{g}^{-3} \\
\text { d.b] }\end{array}$} \\
\hline & $\mathrm{VM}$ & $\mathrm{FC}$ & $\begin{array}{c}\text { Ceni } \\
\text { za }\end{array}$ & & \\
\hline \multicolumn{6}{|l|}{ Hojaras } \\
\hline ca & 62.8 & 11.4 & & & \\
\hline molida & 6 & 6 & 15.73 & 10.01 & 14.87 \\
\hline M10- & 69.0 & 10.3 & & & \\
\hline G00 & 4 & 3 & 20.63 & 8.82 & 15.05 \\
\hline M15- & 65.4 & 11.6 & & & \\
\hline G00 & 4 & 3 & 22.93 & 12.49 & 14.85 \\
\hline M20- & 63.9 & 12.4 & & & \\
\hline G00 & 0 & 7 & 23.64 & 17.10 & 14.20 \\
\hline M10- & 72.0 & & & & \\
\hline G05 & 9 & 9.92 & 17.99 & 8.69 & 15.54 \\
\hline M15- & 67.2 & 12.2 & & & \\
\hline G05 & 2 & 4 & 20.55 & 11.57 & 14.86 \\
\hline M20- & 61.6 & 13.2 & & & \\
\hline G05 & 8 & 6 & 25.06 & 16.89 & 13.84 \\
\hline M10- & 68.0 & 13.0 & & & \\
\hline G10 & 4 & 4 & 18.92 & 9.57 & 15.25 \\
\hline M15- & 67.3 & & & & \\
\hline G10 & 2 & 9.11 & 23.57 & 12.33 & 14.49 \\
\hline M20- & 67.4 & 11.4 & & & \\
\hline G10 & 0 & 5 & 21.15 & 17.24 & 14.85 \\
\hline
\end{tabular}

El análisis de reactividad (Figura 3) muestra que con el aumento del contenido de glicerol, se genera un hombro a una temperatura aproximada de 150 ${ }^{\circ} \mathrm{C}$, la degradación de la hemicelulosa se observa entre $250-280{ }^{\circ} \mathrm{C}$, el pico máximo que corresponde a la celulosa se da a $320^{\circ} \mathrm{C}$ y la degradación de la lignina se presenta entre $360-380^{\circ} \mathrm{C}$.

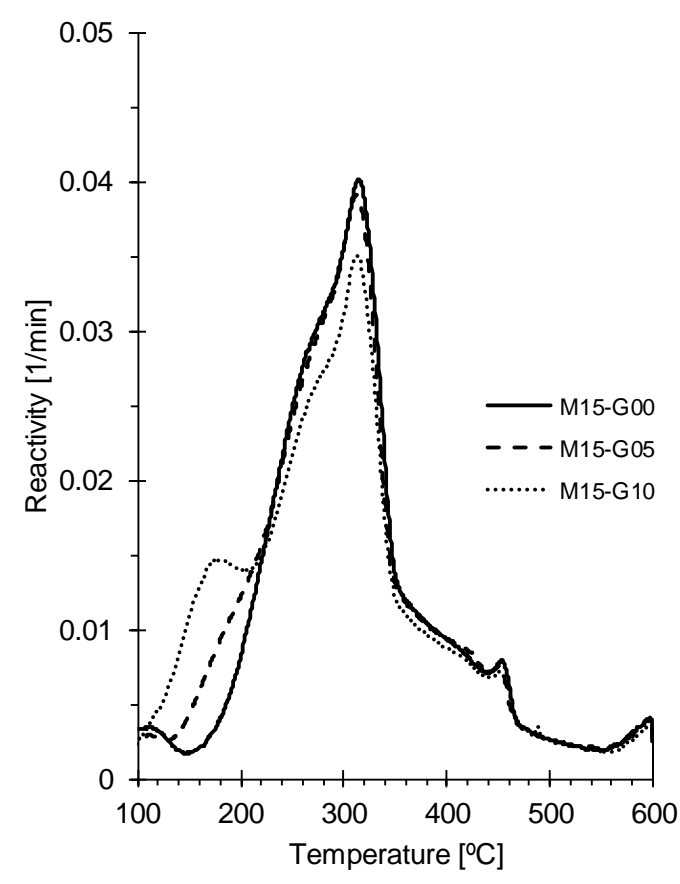

Figura 3. Reactividad

Ésta biomasa $\left(\mathrm{CH}_{1.49} \mathrm{O}_{0.44} \mathrm{~N}_{0.03} \mathrm{~S}\right)$ en promedio posee un poder calorífico inferior en base seca de $13 \mathrm{MJ} / \mathrm{kg}$, estas propiedades hacen viable este material biomásico para ser utilizado en procesos de generación de energía a pequeña escala $(<50$ $\mathrm{kWe}$ ), cuyas plantas están configuradas por sistema de gasificación-motor-generador con una eficiencia global de $15 \%$ a $20 \%$; se estima que una planta operando $8 \mathrm{hr} /$ día usando esta biomasa como combustible renovable tendría un potencial que varía entre $7 \mathrm{kWe}$ y $9 \mathrm{kWe}$ para la Universidad.

\section{CONCLUSIONES}

Las propiedades físicas de la biomasa peletizada evalúan el comportamiento estructural y la calidad del almacenamiento de combustible; además, estos parámetros describen la adaptación y el comportamiento de los pellets utilizados para la recuperación de energía en lechos empacados en procesos termoquímicos. Las propiedades termoquímicas evalúan la calidad del combustible como recurso renovable a partir de los desechos y evaluar los diferentes cambios composicionales que sufre la materia prima al ser tratada en un proceso de peletizado (sometida a presión y temperatura).

\section{AGRADECIMIENTOS}

Los autores agradecen el apoyo financiero a la Universidad de Antioquia a través del proyecto 
CODI "Evaluación energética y emisiones de una estufa eco-eficiente para cocción basada en microgasificación de biomasa a escala domiciliaria. PRG2017-16230”.

\section{REFERENCIAS}

Andersen, J. K., Boldrin, A., Christensen, T. H., \& Scheutz, C. (2010). Greenhouse gas emissions from home composting of organic household waste. Waste Management, 30(12), 2475-2482. http://doi.org/10.1016/j.wasman.2010.07.004

Bhange, V. P., Prince, S. P. M., Vaidya, A. N., \& Chokhandre, A. R. (2012). Green Waste As a Resource for Value Added Product Generation : A Review, 4(1), 22-33.

C. J. Torres, J. F. Archila, M. L. Tronco, M. Becker, A. J. V. Porto, A. J. Tiberti. (2013). Estudio cinemático de una plataforma robótica para agricultura. REVISTA COLOMBIANA DE TECNOLOGÍAS DE AVANZADA, ISSN: 1692-7257. 2(22).

Criollo, B., Alvarado, J., \& Numpaque, H. (2014). PID temperature control and ph dosage for methane. Revista Colombiana de Tecnologías de Avanzada, 2, 134-141.

F. A.L. González, M. M. H. Cely. (2013). Estudio del potencial eólico y solar de Cúcuta, Norte de Santander. REVISTA COLOMBIANA DE TECNOLOGÍAS DE AVANZADA, ISSN: 1692-7257. 2(22).

García-Maraver, A., Zamorano, M., Ramos-Ridao, A., \& Díaz, L. F. (2012). Analysis of olive grove residual biomass potential for electric and thermal energy generation in Andalusia (Spain). Renewable and Sustainable Energy Reviews, 16(1), 745-751.

García, J., Arévalo, J., \& Guerrero, G. (2015). Exergy analysis for a furnace in the production of panela. Revista Colombiana de Tecnologías de Avanzada, 1, 32-39.

Larochelle, L., Turner, M., \& LaGiglia, M. (2012). Evaluation of NAMA opprtunities in Colombia's solid waste sector. Washington.

Leal González, F., \& Hernandez Cely, M. (2013). Estudio del potencial eólico y solar de Cúcuta, Norte de Santander. REVISTA COLOMBIANA DE TECNOLOGÍAS DE AVANZADA, 2(22).

Medina Jimenez, A. C., Nordi, G. H., Palacios Bereche, M. C., Bereche, R. P., Gallego, A. G., \& Nebra, S. A. (2017). Evaluation of two different alternatives of energy recovery from municipal solid waste in Brazil. Waste Management \& Research, 1-12.
Piepenschneider, M., Bühle, L., \& Wachendorf, M. (2016). Solid Fuel Generation from Urban Leaf Litter in Mixture with Grass Cuttings: Chemical Composition, Energetic Characteristics, and Impact of Preprocessing. BioEnergy Research, 9(1), 57-66. http://doi.org/10.1007/s12155-015-9661-7

Torres, C., Archila, J., Tronco, M., Becker, M., Viera Porto, A., \& Tiberti, A. (2013). Estudio cinemático de una plataforma robótica para agricultura. REVISTA COLOMBIANA DE TECNOLOGÍAS DE AVANZADA, 2(22).

William, S. P. M. P., Bhange, V. P., William, S. P. M. P., Suke, S. G., \& Vaidya, A. N. (2015). Quantification and evaluation of garden biomass in municipal solid waste of Nagpur city: A case study, 14(2), 326-331.

Zhang, J., Smith, K. ., Ma, Y., Ye, S., Jiang, F., Qi, W., ... Thorneloe, S. . (2000). Greenhouse gases and other airborne pollutants from household stoves in China: a database for emission factors. Atmospheric Environment, 34(26), 4537-4549. 